\title{
Analysis of the Antenna's Setup Errors at the Global Navigation Satellite System Measurements
}

\author{
Evangelia Lambrou \\ School of Rural and Surveying Engineering, National Technical University of Athens, Athens 15780, Greece
}

\begin{abstract}
Today, the GNSS (global navigation satellite system) is used for more complicate and accurate applications such as monitoring or stake out works. The truth lies in the fact that in the most of the times not enough attention is paid to the antenna's setup. Usually, gross errors are found in the antenna's centering, leveling and in the measurement of its height, which are significant. In this paper, a thoroughly analysis of the above mentioned errors is carried out. The influence of these errors in the calculation of the X, Y, Z Cartesian geocentric coordinates and the $\varphi, \lambda, h$ ellipsoid geodetic coordinates of a point $\mathrm{P}$ on the earth's surface, is analyzed and is presented in several diagrams. Also a new convenient method for the accurate measurement of the antenna's height is presented and it is strongly proposed. The conclusions outline the magnitude of these errors and prove the significance of the antenna's proper setup at the accurate GNSS applications.
\end{abstract}

Key words: GNSS antenna's height, centering and leveling errors, GNSS antenna's setup.

\section{Introduction}

The GNSS (global navigation satellite system) measurements are used for the determination of accurate coordinates of points (of the order of some $\mathrm{mm}$ ), which belong to monitoring networks, or to stake out applications [1-3].

It is well known that the human intervention during the measurements is minimum by using the satellite positioning systems. Namely no sighting is needed, as when total stations are used.

However, a receiver must be put at a selected point, the antenna must be centered and leveled properly and the antenna's height must be measured by using a usually simple tape.

Are all the above-mentioned activities carried out with the proper manner? Mistakes, gross or random

Corresponding author: Evangelia Lambrou, assistant professor, research fields: development of methodology for the determination of astronomical coordinates, precise determination of undulation $\mathrm{n}$ of geoid, interconnected systems, e-geodesy, observations via internet, check and calibration of geodetic instruments (geodetic metrology), development of methodologies for precise measurements, geometric documentation of technical and natural structures. E-mail: litsal@central.ntua.gr. errors, which could be done, when a GNSS antenna placed will be proved significant. Today the minimum uncertainties, which are expected, require major attention to the measurement of antenna's height and to the antenna's centering and leveling in order to acquire correct measurements.

In the following paragraphs, the aforementioned errors are analyzed, in order to determine the magnitude of the error that each one adds in the calculation of the geodetic coordinates $\varphi, \lambda$ and $h$ or the geocentric coordinates $\mathrm{X}, \mathrm{Y}, \mathrm{Z}$ of a point.

As the errors that will be discussed are of the order of some centimeters, it may be considered that the earth is a solid sphere with radius $\mathrm{R}$. This admission is adequate for the analysis.

\section{The Antenna's Height}

As the ellipsoid is the reference surface of the GNSS system, is useful to remind that the height of the antenna $\left(a_{h}\right)$ is measured along the plumb line instead of the geodetic normal as ought to be measured (Fig. 1).

Eq. (1) gives the correct antenna's height: 


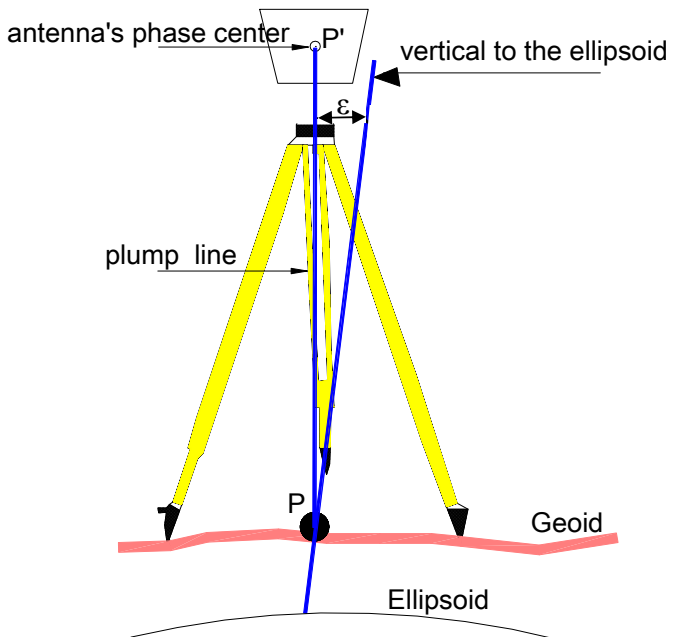

Fig. 1 The measurement of the antenna's height along the plumb line.

$$
a_{h}=a_{h(\text { measured })} \cdot \cos \varepsilon
$$

where, $\varepsilon$ is the deviation of the vertical, at the specific point.

As the deviation of the vertical $(\varepsilon)$ is of about some arcsec and a mean antenna's height is of about $1.5 \mathrm{~m}$, it's obvious that this correction is insignificant.

Thus, when the coordinates of a point $P$ on the earth's surface, $\left(X_{P}, Y_{P}, Z_{P}\right)$ are needed the coordinates at a point $P^{\prime},\left(X_{P^{\prime}}, Y_{P^{\prime}}, Z_{P^{\prime}}\right)$ (Fig. 1) are initially calculated. The point $P^{\prime}$ corresponds to the electric phase center of the antenna.

Using the fundamental equations for the transformation of $\varphi, \lambda$ coordinates to $X, Y, Z$ [4] but simplify them considering that the earth is a sphere with radius $\mathrm{R}$, then the coordinates of the point $P^{\prime}$ can be calculated:

$$
\begin{gathered}
X_{p^{\prime}}=\left(R+h_{p^{\prime}}\right) \cdot \cos \varphi \cdot \cos \lambda \\
Y_{p^{\prime}}=\left(R+h_{p^{\prime}}\right) \cdot \cos \varphi \cdot \sin \lambda \\
Z_{p^{\prime}}=\left(R+h_{p^{\prime}}\right) \cdot \sin \varphi
\end{gathered}
$$

It is pointed out that the geometric height $h$ of $P^{\prime}$ includes the antenna's height measurement $a_{h}$ :

$$
h_{p^{\prime}}=h_{p}+a_{h}
$$

Namely, the component of the antenna's height $a_{h}$ in relation to the geocentric coordinate system $X, Y, Z$ ought to be subtracted from the coordinates $X_{P^{\prime}}, Y_{P^{\prime}}$, $Z_{P^{\prime}}$ in order to the desired coordinates $X_{P}, Y_{P}, Z_{P}$ of the point $P$ be calculated by combining Eqs. (2) and (3).
Assuming that the error of the antenna's height measurement is $e$, then the coordinates of point $P$ will be:

$$
\begin{gathered}
X_{p}=X_{p^{\prime}}-\left(a_{h} \pm e\right) \cdot \cos \varphi \cdot \cos \lambda \\
Y_{p}=Y_{p^{\prime}}-\left(a_{h} \pm e\right) \cdot \cos \varphi \cdot \sin \lambda \\
Z_{p}=Z_{p^{\prime}}-\left(a_{h} \pm e\right) \cdot \sin \varphi
\end{gathered}
$$

The errors $m_{X_{p}}, m_{Y_{p}}, m_{Z_{p}}$, that correspond to the coordinates $X_{P}, Y_{P}, Z_{P}$, due to the error $e$ are as follows:

$$
\begin{gathered}
m_{X_{p}}= \pm e \cdot \cos \varphi \cdot \cos \lambda \\
m_{Y_{p}}= \pm e \cdot \cos \varphi \cdot \sin \lambda \\
m_{Z_{p}}= \pm e \cdot \sin \varphi
\end{gathered}
$$

The magnitude of each component depends on the position of the point $P$ on the earth's surface, namely, its approximate latitude and longitude.

It is noted that, this error does not influence the ellipsoid coordinates $\varphi, \lambda$ of point $P$ but only the geometric height $h$, which bears the total error.

Figs. 2-4 illustrate the change of the components $m_{X_{p}}, m_{Y_{p}}, m_{Z_{p}}$, for an error e equal to $1 \mathrm{~cm}$ in relation to the position $\varphi, \lambda$ of the point $P$ on the earth's surface.

As it is depicted in the diagrams, $m_{X_{p}}$ and $m_{Y_{p}}$ fluctuate from $0 \mathrm{~mm}$ to $10 \mathrm{~mm}$. Their absolute values are minimized at $\varphi= \pm 90^{\circ}$ at the earth's poles and they are maximized near the equator. Also $m_{X_{p}}$ decreases, when $\lambda$ increases as $m_{Y_{p}}$ rises. On the contrary $m_{Z_{p}}$, becomes 0 at the equator and it maximizes at the poles.

\section{Accurate Measurement of the Antenna's Height}

The measurement of the antenna's height using a tape, while it is on a tripod or a pillar is not an accurate procedure. So, some manufacturer are accompanied their receivers with a special equipment for the antenna's height measurements [5]. Also guidelines are given for the proper measurement of an antenna's height $[6,7]$.

A mistake of some millimeters or a little more can be very easily read. Additionally, the proper positioning of the tape on the antenna's hook or on the 


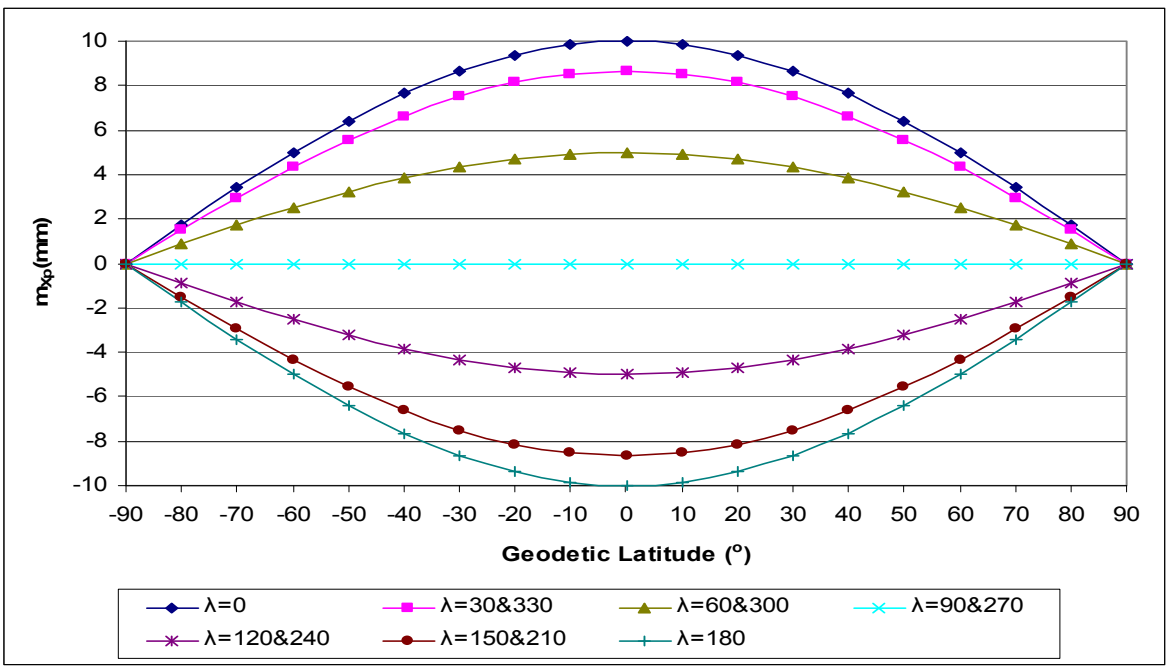

Fig. 2 The error $m_{X_{p}}$ for $\boldsymbol{e}=1 \mathrm{~cm}$.

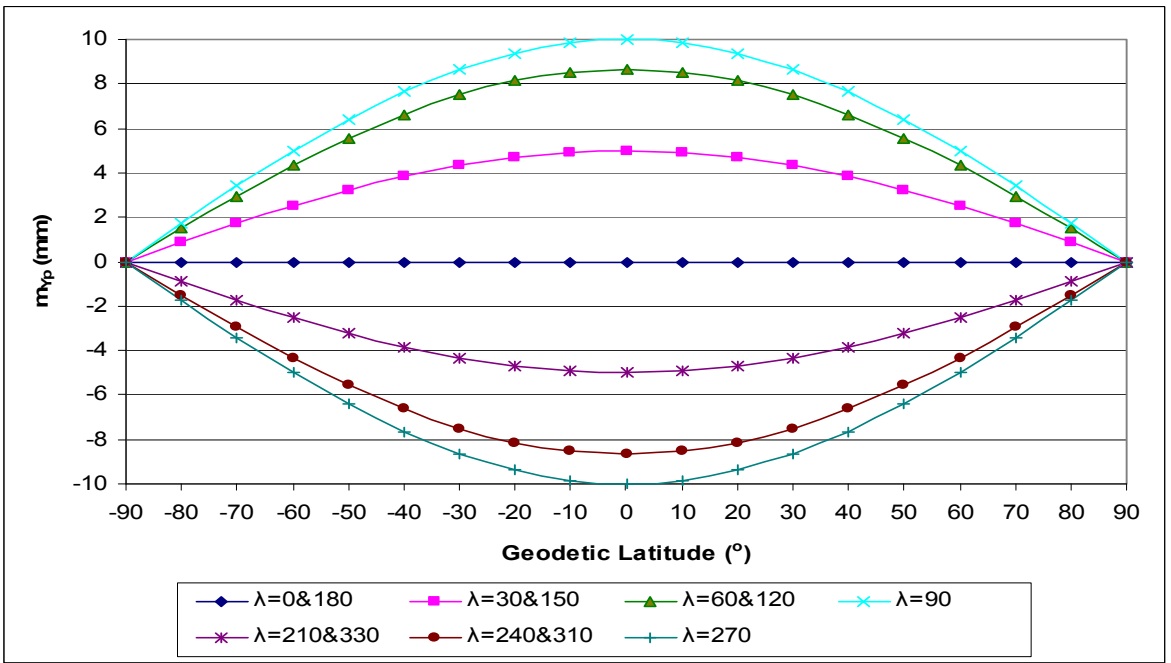

Fig. 3 The error $m_{Y_{p}}$ for $\boldsymbol{e}=\mathbf{1} \mathrm{cm}$.

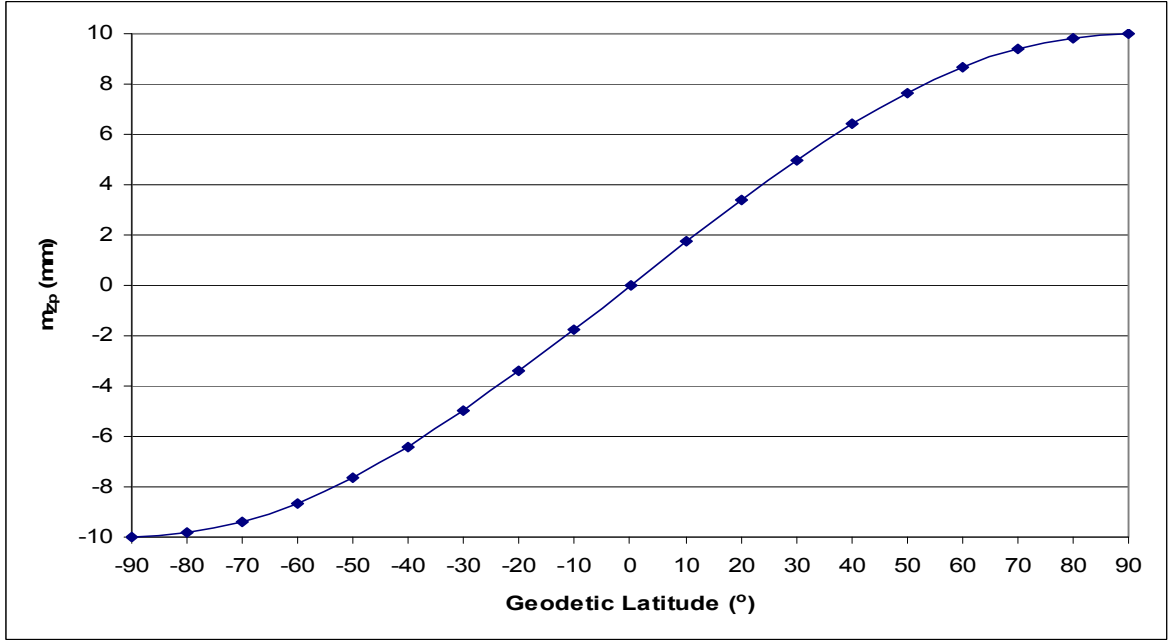

Fig. 4 The error $m_{Z_{p}}$ for $\boldsymbol{e}=1 \mathrm{~cm}$. 
antenna's band or on the antenna's bumper is doubtful. Moreover, the zero point of the tape, most of the times, is not in the proper position. Finally, the misreading of the tape must be taken into consideration as a gross error.

Therefore, for special GNSS applications which need the maximum accuracy, it is proposed that the measurement of the antenna's height should be carried out by using the spirit leveling method as follows:

(1) Before the antenna's setup at a selected point $P$, it is essential for a staff to be put on it (Fig. 5a);

(2) A digital level is placed and is leveled at a close distance of about 3-5 m. The indication $i$ on the staff is then registered;

(3) Next, the tripod with the tribrach and the adaptation base of the antenna is placed, centered and leveled at point $P$;

(4) The staff is put on the surface of the adaptation base where the antenna will be mounted, and a second indication $j$ is registered (Fig. $5 \mathrm{~b}$ ).

The antenna's height $a_{h}$ is calculated easily by the equation:

$$
a_{h}=i-j
$$

By using the above procedure, the true vertical measurement of the antenna's height $a_{h}$ from the bottom of its mount is calculated. It is also indicative that the distance $d$ between the antenna's electric phase center and the bottom of the antenna's mount, is accurately known $\left(m_{d}= \pm 0.1 \mathrm{~mm}\right)$ as it is given by the manufacturers in order to be used in the process of calculating the measurements.

The accuracy that $i$ and $j$ can be measured is of the order of $m_{i}=m_{j}= \pm 0.1 \mathrm{~mm}$ by using a system digital level-barcode staff. Thus, applying the variance covariance low in Eq. (6), the total uncertainty of an antenna's height measurement is determined by:

$$
m_{a_{h}} \pm \sqrt{m_{i}^{2}+m_{j}^{2}}= \pm 0.15 \mathrm{~mm} \approx \pm 0.2 \mathrm{~mm}
$$

The above analyzed procedure proved easy to apply and extremely accurate as it required less than $5 \mathrm{~min}$ to be implemented and protects against gross or systematic tape-reading errors.

\section{The Centering and Leveling Errors}

The centering error is defined as the deviation of the projection along the plumb line of the antenna's phase center from the desire point $P$ (Fig. 6). In correspondence to the centering error, the leveling error has the same definition due to the wrong leveling of the antenna.

Moreover, in this case, the measurement of the antenna's height holds an additional error. These errors present the deviation of the main vertical axis of the antenna, from the plumb line, which intersects the desire point $P$.

Despite the human carelessness during the set up of the antenna, these errors are also caused either due to a tribrach bad check or to a complete overlook of a required check.

The antenna's centering and leveling errors both exist on a horizontal plane, which is perpendicular to the plumb line at point $P$. Both are gross errors and it is difficult to discover and define the true vector, which represent them.

The result of these errors is a vector $P P^{\prime \prime}$ with an unknown absolute value. This vector $e_{c l}$ (Fig. 6) starts from the desired point $P$ and has a random geodetic azimuth $A$.

Considering that the earth is a solid sphere with a mean radius $R=6,371 \mathrm{~km}$ then this vector can be analyzed in two components, one along the meridian, namely $\sigma_{\varphi}$, and the other along the parallel, namely $\sigma_{\lambda}$. The components $\sigma_{\varphi}$ and $\sigma_{\lambda}$, as presented in Fig. 6, are:

$$
\begin{array}{ccc}
\sigma_{\varphi}(m)=e_{c l} \cdot \cos A & \text { or } & \sigma_{\varphi^{\prime \prime}}=\left(\frac{e_{c l} \cdot \cos A}{R}\right) \cdot \rho^{\prime \prime} \\
\sigma_{\lambda}(m)=e_{c l} \cdot \sin A & \text { or } & \sigma_{\lambda^{\prime \prime}}=\left(\frac{e_{c l} \cdot \sin A}{R \cdot \cos \varphi}\right) \cdot \rho^{\prime \prime}
\end{array}
$$

where,

$A$ is geodetic azimuth of the vector $P P^{\prime \prime}\left(e_{c l}\right)$;

$e_{c l}$ is absolute value of the error;

$\rho^{\prime \prime}$ is 206,265 .

The $\sigma_{\varphi}$ fluctuates from $e_{c l}$, if $A=0$ or $A=\pi$, to zero if $A=\pi / 2$ or $A=3 \pi / 2$. The opposite is valid for $\sigma_{\lambda}$. 
(a)

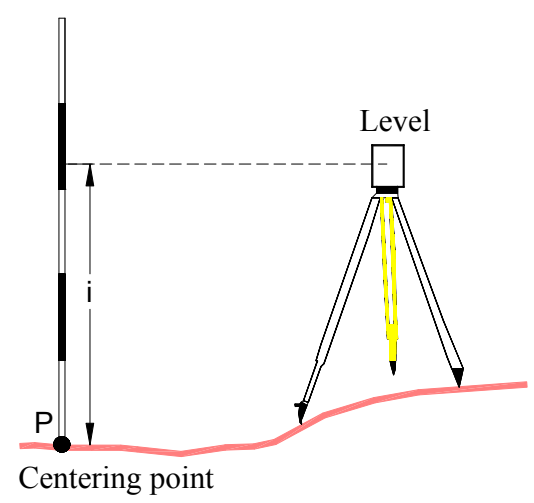

Fig. 5 The accurate measurement of an antenna's height.

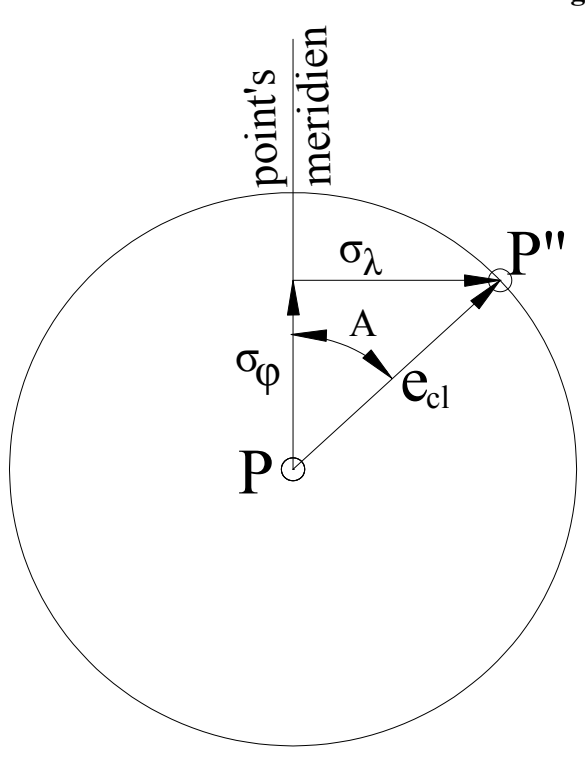

(b)

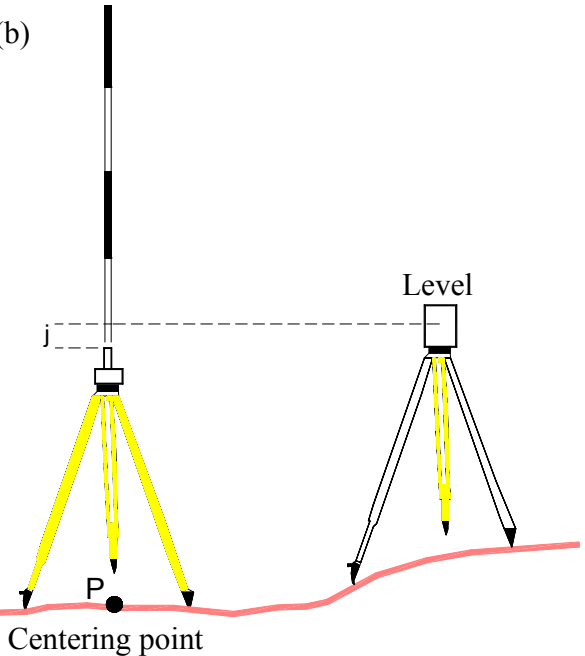

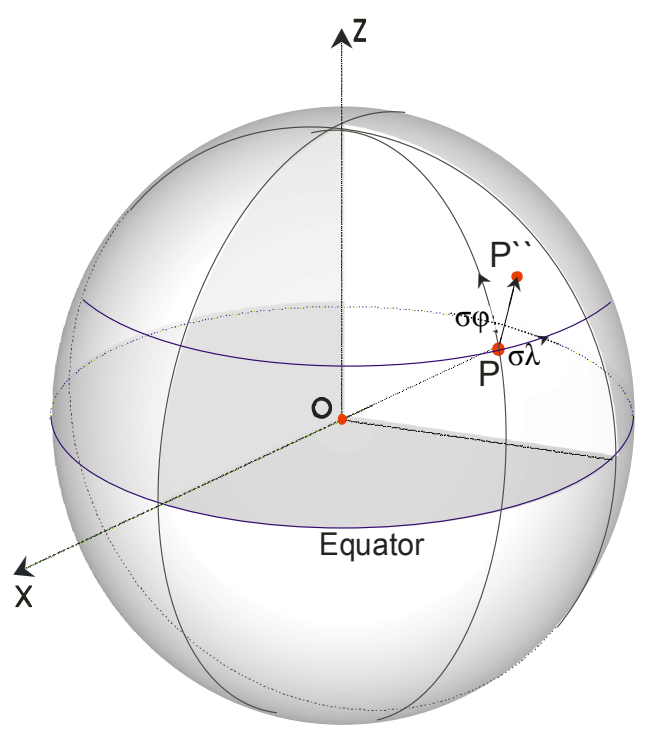

Fig. 6 The analysis of the vector of centering and leveling error.

As previously mentioned, it is difficult to determine the azimuth $A$ of this vector which varies from 0 to $2 \pi$. Thus the mean value $\mathrm{m}$ of this error $e_{c l}$ (centering and leveling) along the meridian according to the Eq. (8) can be calculated as:

$$
m^{2}=\frac{\sum\left(e_{c l} \cdot \cos A\right)^{2}}{n}
$$

where, $n$ is the elementary step dA of azimuth A from 0 to $2 \pi$.

$$
n=\frac{2 \pi}{d A}
$$

According to Eq. (11) the following integral can be formed:

$$
m^{2}=\frac{1}{2 \pi} \int_{0}^{2 \pi}\left(e_{c l} \cdot \cos A\right)^{2} d A
$$

By solving the integral of Eq. (12), the following result comes out:

$$
\begin{gathered}
m^{2}=\frac{1}{2 \pi} \int_{0}^{2 \pi}\left(e_{c l} \cdot \cos A\right)^{2} d A= \\
\frac{1}{2 \pi} \cdot e_{c l}^{2} \cdot \int_{0}^{2 \pi} \cos ^{2} A d A=\frac{1}{2 \pi} \cdot e_{c l}^{2} \cdot \int_{0}^{2 \pi} \frac{\cos 2 A}{2} d A \\
=\frac{1}{2 \pi} \cdot e_{c l}^{2} \cdot\left[\frac{1}{2} \int_{0}^{2 \pi} d A+\frac{1}{2} \int_{0}^{2 \pi} \cos 2 A d A\right]=\frac{e_{c l}^{2}}{2}
\end{gathered}
$$

The same result arises if Eq. (9) used for the calculation of the mean error along the parallel. Thus a mean value of centering and leveling error is 
equal to:

$$
m= \pm \frac{e_{c l}}{\sqrt{2}}
$$

The following chart (Fig. 7) depicts the mean error at $\varphi$ and $\lambda$ due to an error $e_{c l}$ of the antenna's centering and leveling.

This error can also be analyzed in the geocentric coordinates system according to the Eq. (2). It adds an error $m_{c l}$ in the geocentric coordinates $X_{P}, Y_{P}, Z_{P}$ of the desired point $P$ as follows:

$$
\begin{aligned}
m_{c I X p}= & \left(R+h_{p}\right) \cdot\left(\cos \varphi \cdot \cos \lambda-\cos \left(\varphi \pm \sigma_{\varphi}\right) \cdot \cos \left(\lambda \pm \sigma_{\lambda}\right)\right) \\
m_{c l Y p}= & \left(R+h_{p}\right) \cdot\left(\cos \varphi \cdot \sin \lambda-\cos \left(\varphi \pm \sigma_{\varphi}\right) \cdot \sin \left(\lambda \pm \sigma_{\lambda}\right)\right) \\
& m_{c l Z p}=\left(R+h_{p}\right) \cdot\left(\sin \varphi-\sin \left(\varphi \pm \sigma_{\varphi}\right)\right)
\end{aligned}
$$

Consequently, $m_{c I X p}, m_{c l Y p}, m_{c l Z p}$, are the corrections that should be applied to the calculated geocentric coordinates at $P^{\prime \prime}$ in order to achieve the correct coordinates at point $P$. It is pointed out that $\sigma_{\lambda}$ does not add error to the $Z$ coordinate of point $P$. Also the accurate value $h_{P}$ is insignificant compared to the earth's radius for the calculation of the errors $m_{c l}$ by using Eq. (15).

Figs. 8 and 9 illustrate the $m_{c l X p}, m_{c l Y p}$, for an error $\sigma_{\varphi}=\sigma_{\lambda}=1 \mathrm{~cm} \approx 0 "$.0003 for all combinations of $\varphi$ and $\lambda$, assuming that $h_{P}=0$.

Fig. 10 illustrates the $m_{c I Z p}$ for an error $\sigma_{\varphi}=\sigma_{\lambda}=$ $1 \mathrm{~cm} \approx 0 "{ }^{\prime \prime} .0003$ and $\sigma_{\varphi}=\sigma_{\lambda}=5 \mathrm{~cm} \approx 0 "$ ".0015, for all combinations of $\varphi$ and $\lambda$, assuming that $h_{P}=0$.

Figs. 8 and 9 look like nomograms as they are enough complicated. The error $m_{c l}$ takes zero value as well as the maximum value $10 \mathrm{~mm}$, for every longitude but for different latitudes.

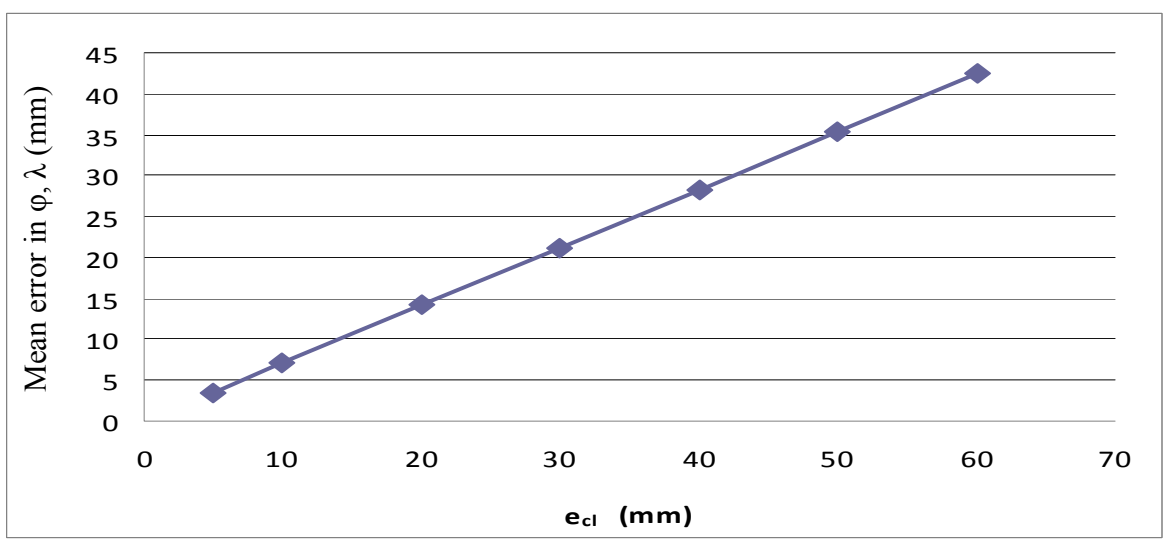

Fig. 7 The mean error at $\varphi$ and $\lambda$ in $\mathbf{m m}$.

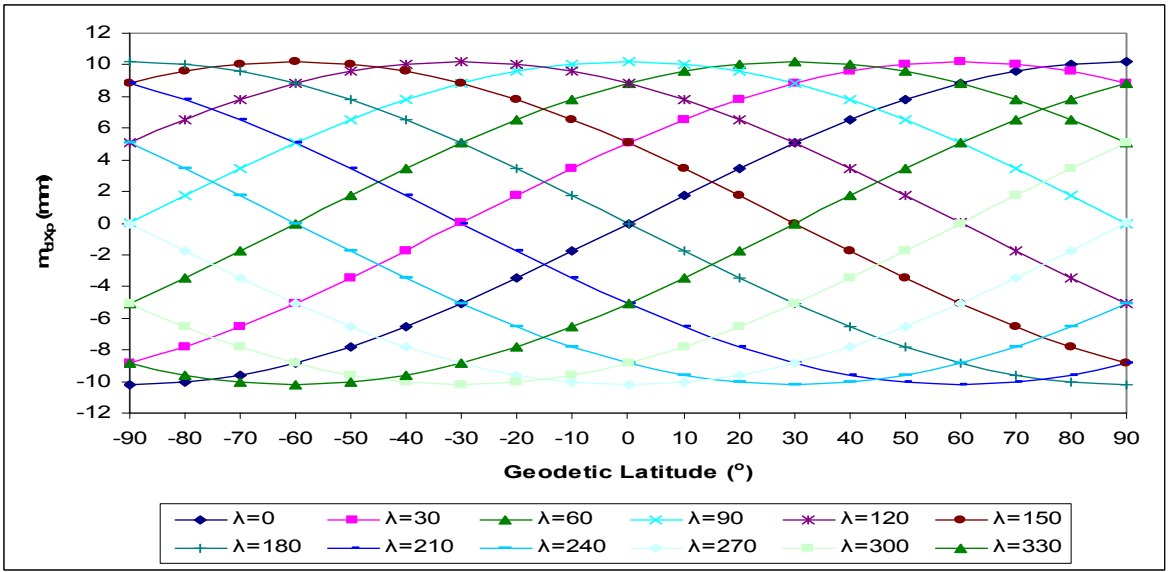

Fig. 8 The $m_{c l X p}$ for an error $\sigma_{\varphi}=\sigma_{\lambda}=1 \mathrm{~cm} \approx 0 " .0003$. 


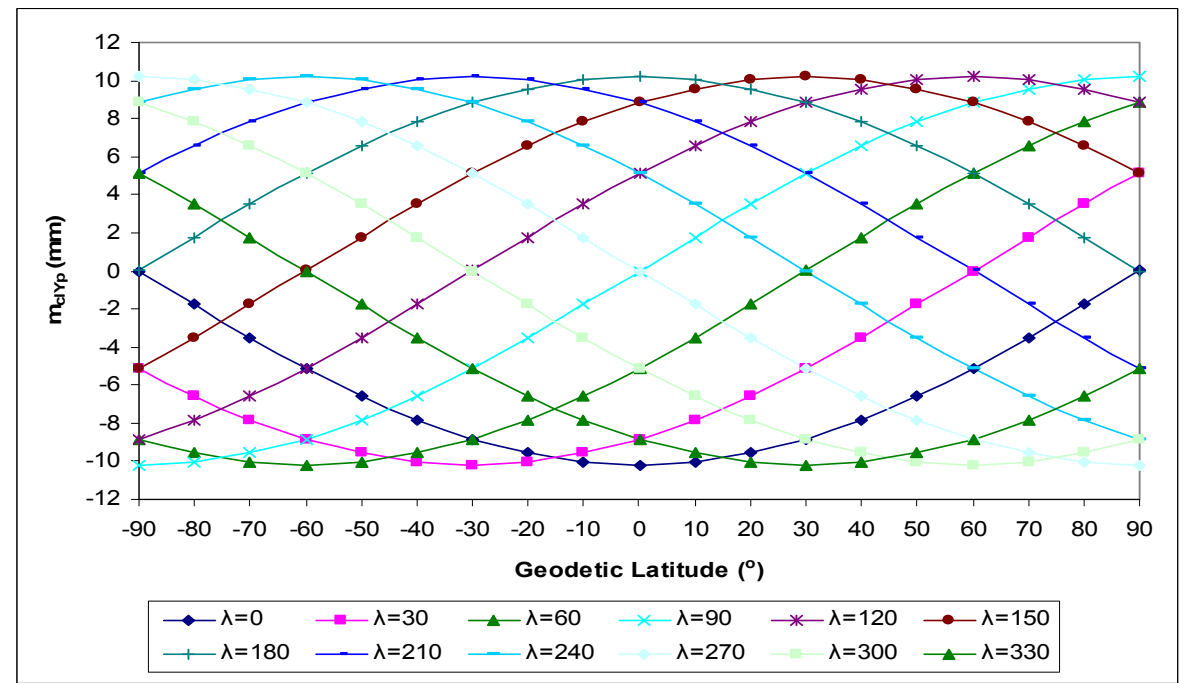

Fig. 9 The $m_{c l Y p}$ for an error $\sigma_{\varphi}=\sigma_{\lambda}=1 \mathrm{~cm} \approx 0 " .0003$.

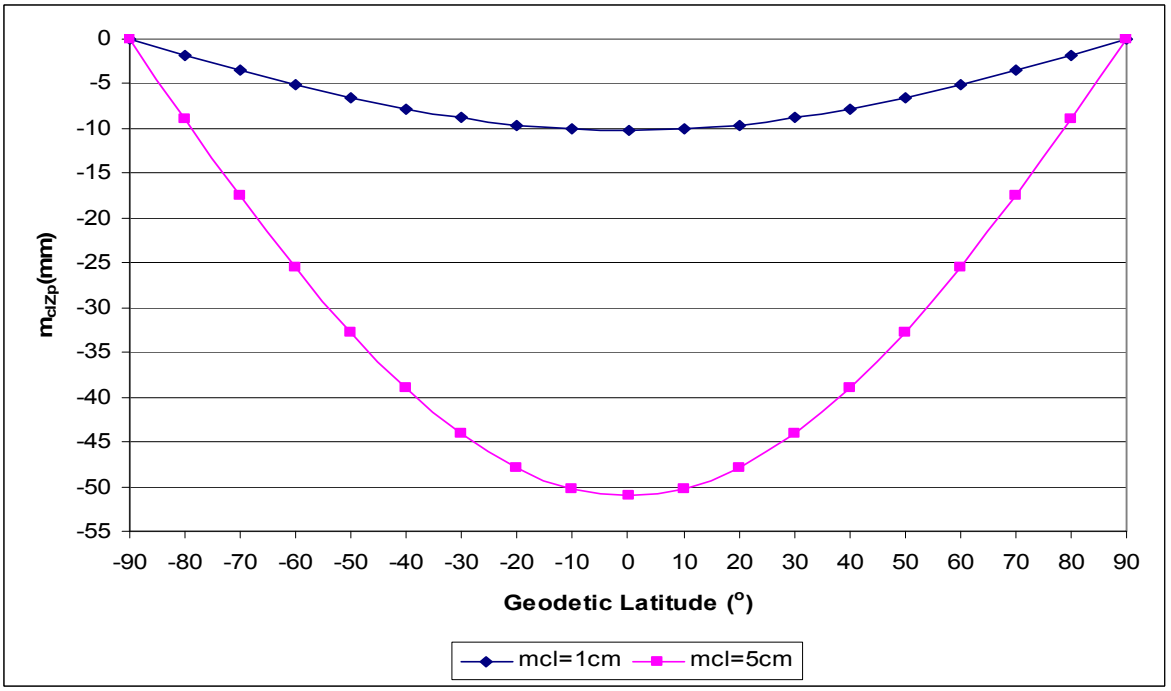

Fig. 10 The $m_{c l Z p}$ for an error $\sigma_{\varphi}=\sigma_{\lambda}=1 \mathrm{~cm} \approx 0 " .0003$ and $\sigma_{\varphi}=\sigma_{\lambda}=5 \mathrm{~cm} \approx 0 " .0015$.

\section{Conclusions}

The inaccuracies of the antenna's setup are disregarded, as no works on this subject were carried out worldwide. Nevertheless, it is proven by the aforementioned analysis, that significant error may change the final coordinates, which are calculated by the GNSS systems.

An error in the antenna's setup, affects the geocentric coordinates $X, Y, Z$, the ellipsoid coordinates $\varphi, \lambda$, and the $h$ of a point $P$.

The transmitted error $e$ in the measurement of the antenna's height at the $X, Y, Z$ geocentric coordinates depends on the position of the point on the earth's surface, namely its latitude and longitude. So, this error in each coordinate fluctuates from 0 to the maximum value $e$.

The error in the measurement of an antenna's height does not affect the $\varphi, \lambda$ coordinates but only the geometric height $h$ of the point.

To avoid this error, a special prototype procedure for the measurement of antenna's height is proposed in order to eliminate this error to $\pm 0.2 \mathrm{~mm}$. This method is efficient, accurate and easy to perform. It is evaluated as worthy, despite the fact that more instrumentation is needed.

The centering and leveling error $e_{c l}$ needs more 
caution as it affects both the geocentric and the ellipsoid coordinates. This error has a mean value of about $\frac{e_{c l}}{\sqrt{2}}$, which is significant for many applications. The real magnitude and the azimuth of the $e_{c l}$ is very difficult and almost impossible to be known. Therefore, careful laboratorial checks of the tribrach should be carried out before each campaign. Otherwise, special manufactured pillars or bases for precise centering should be used in order to eliminate this error at the level of $\pm 0.1 \mathrm{~mm}$.

The above analysis is significant for monitoring networks or infrastructure stake-outs, as the uncertainty of the calculated coordinates by the GNSS may be largely augmented.

\section{References}

[1] M.S. Rawat, V. Joshi, B.S. Rawat, K. Kumar, Landslide movement monitoring using GPS technology: A case study of Bakthang landslide, Gangtok, East Sikkim, India Journal of Development and Agricultural Economics 3 (5) (2011) 194-200.

[2] J.F. Zumberge, M.B. Heflin, D.C. Jefferson, M.M. Watkins, F.H. Webb, Precise point positioning for the efficient and robust analysis of GPS data from large networks, Journal of Geophysical Research: Solid Earth 102 (2012) 5005-5017.

[3] S. Shimada, Y. Bock, Crustal deformation measurements in central Japan determined by a global positioning system fixed-point network, Journal of Geophysical Research: Solid Earth 97 (1992) 437-455.

[4] G. Bomford, Geodesy, 4th ed., Clarendon Press, Oxford, 1980.

[5] Leica Geosystems Solutions Centers Website, http://www.leica-geosystemssolutionscenters.com/Site/In strument\%20PDF's/GPS\%20Systems/Viva/VivaGNSS_E quipList.pdf (accessed Jan. 1, 2013).

[6] D.E. Wells, W. Beck, D. Delikaraoglou, A. Kleusberg, E.J. Krakiwsky, G. Laschappele, et al., Guide to GPS Positioning, University of New Brunswick, Fredericton, New Brunswick, Canada, 1986.

[7] A. Fotiou, C. Pikridas, GPS and Geodetic Applications, Ziti Publications, Thessaloniki, Greece, 2006. 